Revista de Psicología de la PUCP. Vol. XVIII, 1, 2000.

\title{
Estrategias asistenciales e investigación sobre conductas violentas en niños entre 6 y 12 años
}

\author{
Sara Slapak ${ }^{1}$, Nélida Cervone, Ana María Luzzi, Alicia Passalacqua ${ }^{2}$, Norma \\ Menestrina, Teresa Simonotto, María Padawer, Laura Ramos, María Victoria \\ Rodríguez Núñez; Ana María Núñez \\ Universidad de Buenos Aires
}

\begin{abstract}
Se describen las actividades de un Servicio de Psicología Clínica de Niños, las características demográficas y psicopatológicas de la población asistida y se informan resultados del trabajo de investigación realizado en colaboración entre dos cátedras de la Facultad de Psicología de la U.B.A. Se analizaron los protocolos de diagnóstico de 75 niños entre 6 y 12 años de edad que reciben asistencia psicoterapéutica en el Servicio, derivados por escuelas y juzgados en razón de problemas de conducta. Se seleccionaron indicadores que permitan diferenciar las manifestaciones de violencia según respondan a trastornos del carácter, a expresiones de rasgos psicóticos y a síntomas neuróticos como respuestas a situaciones traumáticas del ambiente (situaciones de pérdida). Se buscó, asimismo, detectar indicadores de riesgo suicida en esa población. Los resultados preliminares indican que en la población asistida, proveniente de hogares carenciales de sectores populares del Gran Buenos Aires, predominan conductas violentas como expresión de trastornos del carácter (trastomo disocial de inicio infantil, DSMIV); en menor proporción se registran las manifestaciones de desestructuraciones psicóticas y de síntomas neuróticos. Se observó riesgo potencial de acting out y de conductas autodestructivas inquietantes en los dos primeros casos. Palabras clave: psicopatología infantil, violencia, riesgo suicida.
\end{abstract}

Violent behavior of children between six and twelve years old. Therapeutic strategies and research This paper describes the activities of a Clinical Psychological Unit and the demographic and psychopathological features of the children assisted there. It also informs about some results of the collaborative research work undertaken by two Chairs. Diagnosis records of 75 children between 6 and 12 years old referred by schools and justice courts because of their behavior problems were analyzed. The children belong to poor families of Greater Buenos Aires. Indicators were selected in order to distinguish when the violent behavior is caused by a character disorder, or it is an expression of psychotic features or whether it $t$ is a neurotic symptom as a result of a trauma (loss situation). Another aim is to find indicators of suicidal risk. The preliminary results are that most of the behavior problems are related to character disorder ("disocial disorder of early beginning" DSM IV). Less predominant are the behavior problems as an expression of psychotic features and as neurotic symptoms. There is a potential risk of acting-out and of worrying self-destructive behavior among the two former groups.

Key words: children psychopathology, violence, suicidal risk.

1. Profesora Asociada de la Segunda Cátedra de Psicoanálisis: Escuela Inglesa. Correo electrónico: sslapak@psi.uba.ar.

2. Profesora Titular de la Cátedra de Rorschach.

3. Los otros co-autores forman parte del equipo de las Cátedras de Psicoanálisis y Rorschach. 

El Servicio de Psicología Clínica de Niños, creado por la Segunda Cátedra de Psicoanálisis: Escuela Inglesa en 1990, concreta uno de los propósitos de la Universidad de Buenos Aires, en el sentido de realizar una práctica social que cumpla con el requisito de ser producto de la experiencia docente y de la investigación.

El encuadre comunitario del Servicio permite articular actividades estrictamente asistenciales, con otras de intercambio y asesoramiento a las instituciones de la comunidad. Los niños son derivados mayoritariamente por escuelas y juzgados de la zona. Con todas esas instituciones se mantiene relación continua por medio de entrevistas periódicas y de intercambio sistemático de informes.

Un altísimo porcentaje de niños presenta serios problemas de conducta en la casa, barrio y escuela: comportamientos destructivos, de oposición a normas escolares, robos, vagabundeo, agresiones verbales y físicas a maestros y compañeros, destrucción de muebles y otros elementos escolares, fugas y mentiras. Los problemas de conducta están vinculados mayoritariamente a la destructividad, siendo ésta mucho más frecuente entre los varones (Sautu, Slapak, Di Virgilio, Luzzi y Martinez Mendoza, 1996).

Se observa que el contexto familiar de los niños es conflictivo en la mayoría de los casos: violencia explícita y por omisión; separaciones de la pareja parental; abandono del niño por parte de uno o ambos progenitores; conductas impulsivas en ambos padres; mudanzas, migraciones; muerte de algún integrante de la familia. Es una población que registra una creciente marginación por exclusión económica, elevado porcentaje de padres desocupados o subocupados, asentamiento de viviendas precarias y necesidades básicas insatisfechas en aumento (Slapak, Cervone y Luzzi, 1998). 
La bibliografía (UNICEF, 1991a, 1991b, 1992; CEPAL, 1994; Kliksberg, 1997; Sautu, Slapak, Di Virgilio, Luzzi y Martinez Mendoza, 1999) subraya que el deterioro de las condiciones de vida familiar, social, económica y cultural incide en el incremento de las llamadas "nuevas" patologías, entre las que se encuentran las conductas violentas de niños en edad escolar.

El porcentaje de niños con conductas violentas para los que se solicita asistencia en el Servicio de Psicología Clínica de Niños, ha ido paulatinamente en aumento, especialmente desde 1992. A partir de 1998 se observa, además, un incremento de la consulta por niños con organización psicótica de la personalidad, aunque mayoritariamente no presentan síntomas francos de psicosis y la derivación es por dificultades en el aprendizaje, apatía y dispersión, o impulsividad y descontrol en el ámbito escolar (Cervone, Luzzi, Slapak y Samaniego, 1999).

Desde la creación del Servicio se decidió poner el énfasis en el trabajo grupal. Consideraciones conceptuales, metodológicas e ideológicas permiten sostener que la psicoterapia psicoanalítica grupal, además de ser un instrumento asistencial idóneo, propicia también el desarrollo de sentimientos de pertenencia, alienta la creación de lazos de solidaridad y favorece la construcción de redes sociales de contención.

Se observa que niños muy carenciales, violentos, con familias expulsoras y rechazantes, pueden beneficiarse también con la psicoterapia psicoanalítica grupal; les proporciona un marco de contención y aceptación de su problemática y un sentimiento de pertenencia, en un período histórico como el actual, de aislamiento y disolución del lazo social (Cervone y Luzzi, 1996).

Otras estrategias asistenciales utilizadas en el Servicio son: grupos de orientación a padres o responsables adultos - que se desarrollan en paralelo a la psicoterapia grupal de los niños -, asambleas y psicoterapias 
psicoanalíticas vinculares, familiares e individuales y sólo se brinda asistencia a la población que no tiene cobertura social.

La solicitud de asistencia debe ser acompañada de un informe de la escuela, juzgado o unidad sanitaria que deriva al niño para su atención. El proceso de admisión y evaluación comienza al momento de solicitud de asistencia y consiste, en primer lugar, en una indagación acerca del motivo de consulta y en la administración, a los responsables adultos, de un instrumento de evaluación epidemiológica, el Child Behaviour CheckList (Ackenbach y Edelbrock, 1983). Esta primera instancia, sin tiempo de espera, fue diseñada para recabar información, transmitir el encuadre de trabajo y para propiciar una comunicación tendente a establecer una vinculación con el Servicio, con el objetivo de prevenir una prematura deserción, que es un factor de riesgo característico de esta población.

La evaluación continúa con una entrevista con los responsables adultos para completar la anamnesis y demás datos de la historia clínica. En la entrevista con el niño se utiliza la Hora de Juego Diagnóstica y se administra un test gráfico (Dibujo Libre). El proceso de admisión y evaluación es acotado con la finalidad de formular en el más breve plazo posible la indicación terapéutica e instrumentarla.

Se comunican a la institución de derivación los resultados de la evaluación y las decisiones adoptadas, para continuar la comunicación y, eventualmente, diseñar estrategias conjuntas frente a situaciones problemáticas.

Cabe destacar que los niños, sus familias y las instituciones de la comunidad subrayan la importancia que tiene para ellos que la actividad clínica de base comunitaria se realice desde la Universidad. Constituye para ellos un punto de referencia que permite la creación o recreación de un clima de valoración y respeto propicio para la contención de las situaciones complejas que atraviesan. 
La actividad asistencial desarrollada ininterrumpidamente, generó la necesidad de investigar sobre los problemas más serios y recurrentes de la población clínica. Las actividades de investigación comenzaron a desarrollarse en forma exploratoria desde el inicio del Servicio, y a partir de 1995 en forma sistemática ${ }^{4}$.

Una de las investigaciones actualmente en curso procura la caracterización psicopatológica de las manifestaciones de violencia de los niños desde el marco conceptual de la Teoría de las Relaciones Objetales, teniendo en cuenta la personalidad y el tipo de defensas y ansiedades predominantes. Con ese propósito se solicitó la colaboración de la Cátedra de Rorschach de la Facultad de Psicología de la Universidad de Buenos Aires, a cargo de la Profesora Alicia Passalacqua.

El objetivo de este artículo es presentar el estudio descriptivo realizado por ambos equipos para sistematizar la información recogida elaborando criterios diagnósticos y pronósticos y para la puesta en común de los marcos teóricos respectivos a los fines de la interpretación de los datos obtenidos a partir de las distintas fuentes de información del proceso de evaluación psicológica ${ }^{5}$.

4. PS 043 "Las situaciones de duelo y las tendencias antisociales en niños. Contención familiar y social" (Programación UBACYT 1995-97). TP 047 "Conductas violentas en los niños en edad escolar" y IP 008 "Pobreza, violencia y rendimiento escolar" (Programación UBACYT 1998-2000), acreditados por la Universidad de Buenos Aires y PICT 1626 "Familia, violencia y rendimiento escolar" (Programación 19982000), acreditado por la Agencia Nacional de Promoción de la Investigación Científica y Tecnológica. Los dos últimos se realizan en colaboración con la cátedra de Metodología y Técnica de la Investigación Social de la Facultad de Ciencias Sociales de la U.B.A., a cargo de la Dra. Ruth Sautu y tienen un componente de transferencia bajo la forma de talleres destinados a docentes y autoridades escolares de las escuelas municipales del Partido de Avellaneda (Pcia. De Buenos Aires), sobre el tema de la violencia y el fracaso escolar.

5. El trabajo colaborativo está basado en los proyectos TP 047 "Conductas violentas de niños en edad escolar" y TP 036 "Evaluación de riesgo suicida en las distintas franjas etáreas de la adolescencia", ambos de la Programación UBACYT 19982000. 


\section{Metodología}

\section{Participantes}

A los fines del estudio se consideró, del total de niños admitidos para su asistencia durante 1998 (107 casos) ${ }^{6}$, aquellos que fueron derivados por problemas de conducta, conformándose así una muestra intencional de 75 casos, la que fue a su vez dividida en tres grupos siguiendo criterios y resultados de investigaciones anteriores acerca de la violencia en niños, basadas también en hipótesis teórico-clínicas del marco conceptual de la Teoría de las Relaciones Objetales.

Grupo A. Incluye a los niños cuyas conductas violentas son manifestaciones de la "tendencia antisocial" (Winnicott, 1956) entendida como la emergencia de impulsos inconscientes, que involucran y compelen a otros - padres, maestros, jueces, terapeutas, etc.- a hacerse cargo de su manejo. Pueden presentar dos orientaciones básicas: el robo y la destructividad. Sus manifestaciones coinciden con la descripción del F91.8 "trastorno disocial de inicio temprano" que aparece en el DSMIV (American Psychiatric Association, 1994).

En la base de la tendencia antisocial hay una buena experiencia temprana que se ha perdido. Esta pérdida se produce en circunstancias en las que el niño cuenta ya con cierto grado de estructuración psíquica, que le posibilita percibirla en relación con una falla ambiental. En caso de persistir la falla ambiental la tendencia antisocial puede organizarse en un trastorno del carácter, que podría devenir en psicopatía al llegar a la adolescencia, si no se dieran las oportunidades para la modificación de la falla originaria. Las manifestaciones de violencia están asociadas en algunos casos a dificultades en el aprendizaje y a una propensión a sufrir accidentes.

6. $N=107$ está conformado por: niños con conductas violentas $(n=75)$; niños con problemas de aprendizaje no asociados a conductas violentas $(n=23)$; niños con seria discapacidad mental $(n=9)$. 
Grupo B. Incluye a los niños cuyas conductas violentas son expresión del funcionamiento de la "parte psicótica de la personalidad" (Bion, 1957). Estos niños poseen un menor grado de estructuración psíquica y los rasgos psicóticos aparecen enmascarados por manifestaciones neuróticas, en especial de tipo obsesivo (Klein, 1952).

En los niños de este grupo no se encuentran síntomas clínicos de psicosis franca en el momento de la consulta. Las manifestaciones de violencia aparecen en forma intempestiva y además, el motivo de consulta suele estar relacionado con dificultades de aprendizaje o dificultades en el desarrollo del pensamiento y de la socialización.

Grupo $C$. Incluye a los niños cuyas conductas violentas son expresión de un psiquismo más evolucionado, donde la agresión es expresión de fantasías de celos, exclusión y rivalidad edípica (Klein, 1952). A diferencia de las manifestaciones de violencia de los niños de los Grupos A y B, éstas no tienen características disruptivas ni requieren imperiosa contención. En este grupo se incluyen los niños cuyas manifestaciones de violencia son reactivas a situaciones traumáticas actuales, especialmente de índole familiar.

En el Cuadro 1 aparece la distribución de la muestra intencional de 75 casos de acuerdo a cada uno de los grupos descritos anteriormente.

Cuadro 1

Composición de la Muestra Intencional

\begin{tabular}{|lccccc|}
\hline & Grupo A & Grupo B & Grupo C & Total \\
\hline Varones & 38 & 12 & 19 & 69 \\
Mujeres & 4 & 1 & 1 & 6 \\
Total de niños & 42 & 13 & 20 & 75 \\
\% Total de niños & 56 & 17.34 & 26.66 & 100 \\
\hline
\end{tabular}




\section{Instrumentos}

- Entrevista a los padres o adultos responsables;

- Hora de Juego Diagnóstica;

- Test de Rorschach.

\section{Selección de indicadores psicopatológicos y procesamiento de datos}

Se elaboró un protocolo traduciendo el modelo teórico a actitudes y conductas verbales, no verbales, lúdicas y gráficas desplegadas en las Horas de Juego Diagnósticas, tomando también en cuenta las pautas elaboradas por un grupo de psicólogos "para orientar el análisis y obtener informaciones generalizadoras” (Efron, Fainberg, Kleiner, Sigal, y Woiskoboinik, 1987).

Asimismo se realizó la clasificación y cómputos de los protocolos Rorschach y la puntuación de los signos suicidas según la Escala de Suicidio de signos Rorschach E.S.P.A. (Passalacqua, Orcoyen y Herrera, 1997) ${ }^{7}$.

Los indicadores seleccionados para el análisis de la Hora de Juego Diagnóstica fueron los siguientes:

\section{Actitudes frente al encuadre de trabajo. Componentes:}

- Comprensión de las consignas (con dificultad; sin dificultad)

- Aceptación de las consignas (total; parcial; no aceptación)

- Actividad (pertinente; poco pertinente; no pertinente)

7. La Escala de Suicidio de signos Rorschach (E.S.P.A.) fue construida sobre la base de la confrontación estadística de protocolos Rorschach de una población de 20 suicidados - a quienes se les había administrado Rorschach poco tiempo antes de cometer el acto suicida - con una muestra de 20 adultos tomada de la población general. Media significativa: 20 puntos con una desviación estándar de 2 puntos. 
- Uso del espacio (adecuado; inadecuado: expansivo - restringido intrusivo - otros)

- Uso del tiempo (adecuado; inadecuado: lentificado - acelerado discontinuo - otros)

- Uso de materiales (propios; ofrecidos)

- Relación con el terapeuta (adecuada; inadecuada: desafiante dependiente - evitativa - retraída - otros).

\section{Modalidad de acercamiento al material. Componentes:}

- Previa estructuración del campo;

- Evacuativa

- Dubitativa

- Evitativa

- Impulsiva

- Retentiva.

\section{Modalidad de utilización del material. Categorías:}

- Adecuada

- Inadecuada (evitativa; bizarra; rígida; estereotipada; destructiva; retentiva; otros).

Caracterización de la actividad (lúdica, gráfica, verbalizaciones). Categorías:

- Sostenimiento de la actividad (comienzo, desarrollo y fin) y finalización con de cierre adecuado

- Sostenimiento de la actividad (comienzo, desarrollo y fin) y finalización sin cierre adecuado

- Obstáculos en el sostenimiento de la actividad (interrumpe y continúa con otra acción dentro de la misma actividad; interrumpe y continúa con otra actividad) y finalización con cierre adecuado

- Obstáculos en el sostenimiento de la actividad (interrumpe y continúa 
con otra acción dentro de la misma actividad; interrumpe y continúa con otra actividad) y finalización sin cierre adecuado

- Interrupción de la actividad y finalización sin cierre.

Actitudes y manifestaciones emocionales predominantes. Se consideraron:

- Indiferencia; interés; angustia; llanto; ira; vergüenza; desafío; humillación; excitación; euforia; alegría; hostilidad; enojo; ansiedad; depresión; tristeza; otros.

\section{Rorschach}

- Localizaciones

- Determinantes

- Contenidos

- Fórmulas

- Fenómenos especiales

\section{Resultados}

Los resultados del estudio descriptivo realizado sobre las Horas de Juego Diagnóstico expresan los porcentajes hallados en cada uno de los indicadores seleccionados para el análisis, para cada uno de los grupos de niños considerados.

Los resultados del análisis de las actitudes frente al encuadre (Cuadro 2) dan cuenta de que los niños del Grupo A son los que presentan los mayores porcentajes de comprensión y aceptación total de las consignas, de pertinencia de la actividad, uso adecuado del espacio y uso adecuado del tiempo pero tienen dificultades en la relación con el entrevistador. Los niños del Grupo B son, en cambio, los que registran las mayores dificultades en todos los componentes del encuadre. Los niños del Grupo $C$ no presentan dificultades en este ítem del protocolo. 


\section{Cuadro 2}

Hora de Juego Diagnóstica: Actitudes Frente al Encuadre

\begin{tabular}{|lrrr|}
\hline & Grupo A & Grupo B & Grupo C \\
\hline & & & \\
\hline Comprensión de las consignas & & & \\
sin dificultad & 95.2 & 69.2 & 95 \\
Aceptación total de las consignas & 81 & 53.8 & 65 \\
Aceptación parcial de las consignas & 11.9 & 30.8 & 25 \\
No aceptación de las consignas & 4.8 & 15.4 & 10 \\
Pertinencia de la actividad & 88.1 & 76.9 & 85 \\
Uso adecuado del espacio & 81 & 46.2 & 80 \\
Uso inadecuado-expansivo del espacio & 9.5 & 23.1 & 10 \\
Uso inadecuado-restringido del espacio & 7.1 & 23.1 & - \\
Uso inadecuado - otro del espacio & - & 7.7 & 10 \\
Uso adecuado del tiempo & 88.1 & 69.2 & 80 \\
Uso inadecuado - lentificado del tiempo & 4.8 & - & - \\
Uso inadecuado - acelerado del tiempo & 2.4 & 7.7 & - \\
Uso inadecuado - discontinuo del tiempo & 2.4 & 23.1 & - \\
Uso inadecuado - otro del tiempo & - & - & 20 \\
Uso de los materiales ofrecidos & 88.1 & 76.9 & 85 \\
Relación adecuada con el entrevistador & 59.5 & 53.8 & 75 \\
Relación inadecuada - desafiante & 11.9 & 15.4 & 10 \\
Relación inadecuada - dependiente & - & - & 5 \\
Relación inadecuada - evitativa & 16.7 & - & 5 \\
Relación inadecuada - retraída & 9.5 & 23.1 & - \\
Relación inadecuada - otra & - & 7.7 & 5 \\
\hline
\end{tabular}

Nota. Caso perdido: uno del grupo A.

El análisis de la modalidad de acercamiento al material (Cuadro 3) da cuenta de que la modalidad de "previa estructuración del campo" predomina en los tres grupos aunque los porcentajes mayores corresponden al Grupo $C$ y, en segundo término, al Grupo A. Son llamativamente altos los porcentajes de la modalidad evitativa e impulsiva de acercamiento al material en el Grupo $B$. 
Estrategias asistenciales e investigación sobre conductas violentas

Cuadro 3

Hora de Juego Diagnóstica: Modalidad de Acercamiento al Material

\begin{tabular}{|lccc|}
\hline & Grupo A & Grupo B & Grupo C \\
\hline & & P o r c e n t a j e & \\
\hline Previa estructuración del campo & 59.5 & 38.5 & 65 \\
Evitativa & 19.1 & 30.8 & 10 \\
Evacuativa & 2.4 & 7.7 & - \\
Impulsiva & 2.4 & 23.1 & - \\
Dubitativa & 7.1 & - & - \\
Retentiva & - & 7.7 & - \\
\hline
\end{tabular}

Nota. Casos perdidos: 4 del grupo A y 5 del grupo C.

El Cuadro 4 contiene los resultados obtenidos en el análisis de la modalidad de utilización del material. La modalidad adecuada predomina en los niños del Grupo A y en los del Grupo $C$, aunque es levemente mayor en este último. Los niños del Grupo $B$ tienen, comparativamente, el porcentaje más alto en la modalidad inadecuada - evitativa y son los únicos niños que registran, en un porcentaje aún más elevado, la modalidad inadecuada - destructiva.

\section{Cuadro 4}

Hora de Juego Diagnóstica: Modalidad de Utilización del Material

\begin{tabular}{|lccc|}
\hline & Grupo A & Grupo B & Grupo C \\
\hline Adecuada & & P r c e n t a j e \\
Inadecuada - evitativa & 64.3 & 46.2 & 65 \\
Inadecuada - destructiva & 14.3 & 15.4 & 10 \\
Inadecuada - retentiva & - & 23.1 & - \\
Inadecuada - otras & 2.4 & - & 5 \\
& 2.4 & 7.7 & - \\
\hline
\end{tabular}

Nota. Casos perdidos: 7 del grupo A, 1 del grupo B y 4 del grupo C. 
Los resultados del análisis de la caracterización de la actividad se observan en el Cuadro 5. Los niños del Grupo $C$ son los que tienen el porcentaje más alto en el componente de sostenimiento y finalización con cierre adecuado de la actividad. Los niños del Grupo B, si bien tienen un porcentaje alto en ese componente, son los que también tienen el porcentaje más alto en el correspondiente a los obstáculos en el sostenimiento sin cierre adecuado. Los niños del Grupo A son los que tienen el porcentaje más bajo en el sostenimiento y finalización con cierre adecuado, pero los correspondientes al sostenimiento y finalización sin cierre adecuado y a los obstáculos en el sostenimiento son menores que los del Grupo B.

\section{Cuadro 5}

Hora de Juego Diagnóstica: Caracterización de la Actividad

\begin{tabular}{|lccc|}
\hline & Grupo A & $\begin{array}{c}\text { Grupo B } \\
\text { P o r c e n t a j e }\end{array}$ & Grupo C \\
\hline $\begin{array}{l}\text { Sostenimiento y finalización } \\
\text { con cierre adecuado }\end{array}$ & 29.3 & 38.5 & 40 \\
$\begin{array}{l}\text { Sostenimiento y finalización } \\
\text { sin cierre adecuado }\end{array}$ & 17.1 & 7.7 & 10 \\
$\begin{array}{l}\text { Obstáculos en el sostenimiento } \\
\text { con cierre adecuado } \\
\text { Obstáculos en el sostenimiento } \\
\text { sin cierre adecuado } \\
\text { Interrupción de la actividad y } \\
\text { finalización sin cierre }\end{array}$ & 14.7 & 15.4 & 5 \\
\hline
\end{tabular}

Nota. Casos perdidos: 5 del grupo A y 5 del grupo C.

El análisis de las actitudes y manifestaciones emocionales (Cuadros 6,7 y 8 ) da como resultado que los niños del Grupo $A$ son los que presentan los porcentajes más altos de interés a lo largo de la Hora de Juego Diagnóstica. Los niños del Grupo B, que tienen los menores porcentajes 
de interés al comienzo de la actividad, los van incrementando, mientras decrecen los correspondientes a la indiferencia. Los niños del Grupo $C$ son los únicos que presentan manifestaciones de tristeza, enojo y ansiedad.

Cuadro 6

Hora de Juego Diagnóstica. Comienzo de la Actividad

\begin{tabular}{|lrrr|}
\hline & Grupo A & $\begin{array}{c}\text { Grupo B } \\
\text { P o r c e n t a j e }\end{array}$ & Grupo C \\
\hline Interés & 64.4 & 15.4 & 55 \\
Vergüenza & 9.6 & 15.4 & 5 \\
Desafío & 9.5 & 7.7 & - \\
Indiferencia & 14.4 & 46.2 & 30 \\
Tristeza & - & - & 5 \\
Excitación & - & - & 5 \\
\hline
\end{tabular}

Cuadro 7

Hora de Juego Diagnóstica. Desarrollo de la Actividad

\begin{tabular}{|lrrr|}
\hline & Grupo A & Grupo B & Grupo C \\
\hline Interés & \multicolumn{2}{c}{ P c e n t a j e } \\
Vergüenza & 76.3 & 61.6 & 65 \\
Desafío & 4.8 & 7.7 & - \\
Indiferencia & - & - & - \\
Tristeza & 9.6 & 15.4 & 25 \\
Excitación & - & - & 5 \\
Angustia & 2.4 & 7.7 & - \\
Enojo & 12 & 7.7 & - \\
& - & - & 5 \\
\hline
\end{tabular}


Cuadro 8

Hora de Juego Diagnóstica. Finalización de la Actividad

\begin{tabular}{|lrrr|}
\hline & Grupo A & Grupo B & Grupo C \\
& & P o r c e n t a j e & \\
\hline Interés & 64.4 & 69.2 & 55 \\
Vergüienza & 4.8 & 7.7 & - \\
Desafío & - & - & - \\
Indiferencia & 14.2 & 7.7 & 20 \\
Tristeza & - & - & $5 \%$ \\
Excitación & 14.4 & 15.4 & - \\
Angustia & 11.9 & - & - \\
Enojo & - & - & 5 \\
Ansiedad & - & - & 15 \\
\hline
\end{tabular}

Nota. Casos perdidos: 5 del grupo A y 5 del grupo C.

Cuadro 9

Protocolos Rorschach. Puntuación de los Signos de Riesgo Suicida (E.S.P.A.)

\begin{tabular}{|lc|}
\hline & Media \\
\hline Grupo A & 12,51 \\
Grupo B & 13,66 \\
Grupo C & 11,88 \\
\hline
\end{tabular}

\section{Discusión y conclusiones}

Este trabajo se circunscribe al análisis de indicadores formales de la Hora de Juego Diagnóstica y a la puntuación de signos suicidas en el test 
de Rorschach. A continuación se presenta la discusión y las conclusiones de los resultados, organizada de acuerdo a los diferentes grupos estudiados.

Para el Grupo A se ratifican resultados de investigaciones anteriores sobre niños con tendencia antisocial ${ }^{8}$. Es el grupo que presenta mayor porcentaje de comprensión y aceptación total de las consignas, de pertinencia de la actividad, uso adecuado del espacio y uso adecuado del tiempo, esto es, de todos los componentes relativos al encuadre (Cuadro 2).

Respecto de la relación con el entrevistador, son niños que muestran actitudes y conductas adecuadas en el primer contacto con él aunque tienen dificultad en el vínculo, anticipando conflictos que aparecerán posteriormente, en el transcurso de las sesiones psicoterapéuticas.

Los indicadores del encuadre señalan un funcionamiento psíquico con un cierto nivel de desarrollo y estructuración. Desde el punto de vista de la Teoría de las Relaciones Objetales, son niños que tienen acceso a la posición depresiva aunque tienen dificultades para sostenerla. Sus actitudes y conductas de adecuación a la realidad y tolerancia a la frustración producen impacto en el entrevistador y se interpretan como el resultado del uso de mecanismos sobreadaptativos de control y dominio sobre los objetos y de postergación de la descarga.

Con respecto de la modalidad de acercamiento al material de la Hora de Juego Diagnóstica (Cuadro 3) predomina la "previa estructuración del campo" que indicaría cierta capacidad de anticipación a la acción, aunque entendida como expresión de una inteligencia "práctica".

En segundo lugar aunque en un porcentaje mucho menor se registra la modalidad "evitativa", que en este grupo de niños no está asociado a defensas neuróticas (del tipo de las defensas fóbicas) sino a mecanismos

8. PS 043 "Las situaciones de duelo y las tendencias antisociales en niños. Contención familiar y social" (Programación UBACYT 1995-97). 
de defensa propios de una organización psíquica que podría devenir en psicopatía de no mediar alguna intervención psicoterapéutica. Dentro de esta clase de modalidad "evitativa" se incluyen conductas tales como rehuir la mirada o mirar de soslayo y actitudes defensivas destinadas a bloquear el compromiso emocional frente a temas y situaciones conflictivas. Estas dificultades, asociadas con las que surgen en la relación con el entrevistador, se interpretan como expresión de defensas maníacas para lidiar con ansiedades persecutorias y depresivas, que pueden dar lugar a una situación transferencial negativa en el decurso de una psicoterapia.

Con relación a la utilización del material (Cuadro 4) hay un predominio de una modalidad adecuada, que da cuenta de un funcionamiento psíquico que admite el principio de realidad. En segundo lugar, en un porcentaje mucho menor, se registra una modalidad de utilización inadecuada - evitativa que, al igual que lo interpretado respecto de la modalidad inadecuada - evitativa de acercamiento al material, se entiende como una defensa destinada a bloquear el compromiso emocional.

En cuanto a la caracterización de la actividad (Cuadro 5), se registra un leve predominio de la capacidad de sostenimiento - desarrollo de secuencias de actividad con comienzo, desarrollo y finalización - por sobre los obstáculos en el sostenimiento y casi no hay diferencias en los porcentajes relativos a la capacidad de cierre. Estos valores, notoriamente más bajos que los correspondientes a las actitudes frente al encuadre, ponen en evidencia la existencia de dificultades en la producción simbólica, que se interpretan como producto del ejercicio de mecanismos de control y dominio que, en tanto formas de la defensa maníaca, son precarios y limitan los logros de la sobreadaptación inicial.

Respecto de las actitudes y manifestaciones emocionales (Cuadros 6,7 y 8 ), el alto porcentaje de interés y el relativamente bajo porcentaje de indiferencia demostrados en la actividad, asociados a los resultados 
de los indicadores ya descritos, se interpretan como la expresión de una necesidad de control sobre el mundo externo vinculado a ansiedades persecutorias. No se trataría de un interés genuino, fundado en el despliegue de una curiosidad orientada a la exploración y experimentación de recursos para el desarrollo de procesos simbólicos. Se trataría en cambio, de un estado de alerta movilizado por ansiedades persecutorias, para la detección de "peligros" que deben ser neutralizados mediante mecanismos de control y dominio. Implica un uso rígido y monótono de los recursos, que no enriquece el desarrollo de los procesos simbólicos.

Llama la atención que, tratándose de niños derivados para su atención por sus problemas de conducta, presenten bajos porcentajes de actitudes de desafío al comienzo de la actividad que además, desaparecen en el decurso de la Hora de Juego Diagnóstica; se pueden entender como expresión de un intento de aplacamiento del objeto.

La presencia de angustia y excitación durante el desarrollo de la actividad y su cierre, inexistentes al comienzo, también indica la precariedad y el fracaso parcial de los recursos defensivos utilizados y, por ende, es un indicio de buen pronóstico; señala que las defensas no se han estructurado todavía como rasgos de carácter, por lo que pueden esperarse cambios importantes durante un proceso psicoterapéutico.

El Grupo B es el que registra mayores dificultades en los indicadores del encuadre (Cuadro 2). Presenta los menores porcentajes en la comprensión y aceptación de consignas, en la pertinencia de la actividad y en el uso adecuado del espacio y del tiempo, dando cuenta de dificultades en el desarrollo de los procesos simbólicos.

También se registran los menores porcentajes de adecuada relación con el entrevistador. Las inadecuaciones se relacionan predominantemente con conductas de retracción y, en menor porcentaje, de desafío y se interpretan como modos primitivos de lidiar con ansiedades, que son exclusivamente persecutorias en este grupo de niños. 
La inadecuación en el uso del espacio, que tiende a ser de uso restringido o expansivo y las dificultades en la relación con el entrevistador se pueden entender por la presencia de ansiedades persecutorias intensas, que interfieren la exploración adecuada de los objetos del mundo externo, necesaria para el desarrollo de actividades lúdicas. Esto indicaría restricciones en la capacidad de simbolización; de hecho, se trata de niños que, además, tienen dificultades de aprendizaje.

La inadecuación en el uso del tiempo, que tiende a ser de uso discontinuo y en menor medida, acelerado, se interpreta como la existencia de mecanismos de escisión y como la expresión de un uso abusivo de las identificaciones proyectivas (Bion, 1957).

En síntesis, las dificultades en todos los indicadores del encuadre se interpretan como carencia de recursos yoicos adaptativos frente al impacto de la situación a afrontar.

En cuanto al acercamiento al material (Cuadro 3), presentan el menor porcentaje en la modalidad de "previa estructuración del campo" anticipatoria de la acción - y los mayores porcentajes en las modalidades "evitativa" e "impulsiva".

En este grupo de niños, la modalidad evitativa no es expresión de defensas neuróticas sino de un retraimiento, en tanto defensa primitiva frente a intensas ansiedades persecutorias, que interfieren la exploración adecuada de los objetos del mundo externo y el establecimiento del principio de realidad.

Asimismo, la impulsividad emerge como consecuencia de las falencias en el contacto con la realidad interna y externa, también en relación con ansiedades persecutorias intensas.

Respecto de la modalidad de utilización del material (Cuadro 4), registran iguales porcentajes de utilización adecuada e inadecuada, aunque 
cabe destacar que, comparativamente, la modalidad de utilización adecuada es baja. Las inadecuaciones son, en primer lugar, destructivas - en correspondencia con la impulsividad registrada en el ítem de modalidad de acercamiento al material - y en segundo término, evitativas, lo que subrayaría las interpretaciones anteriormente formuladas acerca de las dificultades de conexión con los objetos.

En cuanto a la caracterización de la actividad (Cuadro 5), es el grupo que registra los mayores obstáculos para sostenerla, lo que daría cuenta de la precariedad de las defensas utilizadas para lidiar con la ansiedad, que se vincula con el retraimiento mencionado en ítemes anteriores.

Aunque en el cierre de la actividad se registran menores obstáculos, esto no se entiende como expresión de la elaboración de ansiedades depresivas, sino como un automatismo frente a la indicación de finalización de la actividad, ya que simultáneamente se observan - en el ítem correspondiente al uso del tiempo -, actitudes y conductas cuyo análisis permite interpretar que la percepción del paso del tiempo no responde a criterios de realidad.

En cuanto a las actitudes y manifestaciones emocionales (Cuadros 6,7 y 8), es el grupo que presenta menor porcentaje de interés y, consecuentemente, mayor grado de indiferencia al comienzo de la actividad, en correspondencia con las actitudes de retracción ya analizadas. Los porcentajes de interés aumentan y los de indiferencia disminuyen de manera abrupta a lo largo de la Hora de Juego Diagnóstica y esto se interpreta como expresión de la utilización masiva de mecanismos de identificación proyectiva, más que como una genuina conexión con el mundo externo.

Los porcentajes pequeños de vergüenza que, aunque van disminuyendo, están presentes en toda la Hora de Juego, así como los escasos porcentajes de angustia en el desarrollo de la actividad, serían 
las únicas expresiones emocionales del funcionamiento de la parte no psicótica de la personalidad.

La excitación, que se manifiesta en el desarrollo y aumenta con la finalización de la actividad, se asocia a la impulsividad registrada en el ítem de modalidad de acercamiento al material y se interpreta como el desborde de una emocionalidad difusa.

El Grupo C no presenta dificultades en los componentes del encuadre (Cuadro 2). Se registran altos porcentajes en la comprensión de las consignas, en la pertinencia de la actividad, en el uso adecuado del espacio y del tiempo y de los materiales; hay adecuada relación con el entrevistador.

Sin embargo, presentan un porcentaje menor de aceptación total de las consignas respecto del Grupo A, lo que indicaría mayores posibilidades de expresión de sus conflictos emocionales y de su ambivalencia, desde el inicio de la actividad y que son niños que no utilizan mecanismos de sobreadaptación para afrontar la situación de evaluación.

En cuanto a la modalidad de acercamiento al material (Cuadro 3), el mayor porcentaje en la modalidad de "previa estructuración del campo" daría cuenta de posibilidades adaptativas frente a situaciones nuevas, sin el costo de la sobreadaptación. Asimismo, es el grupo que presenta el mayor porcentaje de adecuación en la utilización del material (Cuadro 4), ajustada a criterios de realidad.

En lo que respecta a la caracterización de la actividad (Cuadro 5), es el grupo que mejor puede desarrollar secuencias de actividad, dándoles un cierre adecuado; sólo un porcentaje reducido presenta obstáculos en el sostenimiento de la actividad lúdica.

El análisis de las actitudes y manifestaciones emocionales (Cuadros 6,7 y 8 ) da cuenta de que manifiestan un predominio del interés, que se 
mantiene a lo largo de la Hora de Juego. En la finalización, se observa un tenue descenso al respecto y, simultáneamente, se registra la presencia de tristeza, ansiedad y enojo. Es el único grupo que manifiesta estas emociones, que ponen en evidencia la existencia de ansiedades depresivas y son pertinentes al momento de cierre de la actividad. La adecuación al encuadre, la fluidez en la expresividad y la capacidad de sostener la actividad sin obstáculos relevantes, permiten interpretar que este grupo es el que tiene mayores posibilidades para la elaboración de sus conflictos, porque cuenta con recursos yoicos adecuados y la ansiedad no interfiere en la capacidad para simbolizar los conflictos.

En cuanto a los indicadores de riesgo suicida (Cuadro 9), utilizando la E.S.P.A. (Passalacqua et al, 1997), se registra que la media de signos de riesgo suicida en Rorschach del Grupo $B$ se acerca considerablemente a la obtenida por adolescentes no consultantes, según los resultados de una investigación anterior. Cabe destacar que los adolescentes constituyen una población comprobada de riesgo, dada la similitud de su media de signos suicidas $(13,68)$ con muestras de potencial suicida de adultos $(16,20)^{9}$.

Se produce una disminución de signos en el Grupo $A$ y más aún en el Grupo $C$, aunque resultan elevadas todas las medias, si se comparan con las de adultos no consultantes $(6,9)$, lo que es también alarmante.

Es significativo también, que en el Grupo A más que en el Grupo B, y en éste más que en el Grupo C (en donde sólo hay un caso), algunos casos individualmente alcanzan el puntaje significativo de riesgo (20). Esto indicaría que los niños que tienen manifestaciones de defensas que podrían devenir en psicopáticas, también pueden dirigir la agresión hacia sí mismos, aunque en menor proporción que en los casos de desórdenes graves como son los del Grupo B. En tal sentido, una de las hipótesis con

9. Passalacqua y Casullo: "Comportamiento suicida adolescente: análisis de los principales factores de riesgo" - Programación UBACYT 1995-97). 
respecto a la propensión a los accidentes registrada en las historias clínicas de los niños del Grupo A, sería que, probablemente, en este grupo esa propensión a accidentarse esté asociada al uso de mecanismos maníacos omnipotentes, más que a la presencia de ideación suicida.

Otro aspecto llamativo es que, al realizarse una comparación (Passalacqua et al., 1999) con los niños derivados al Servicio también durante 1998, fundamentalmente por problemas de aprendizaje no asociados con conductas violentas $(N=23)$, su media resultó superior a la de los otros grupos (13.95). Esto parece confirmar que el hecho de que no se exprese explícitamente la violencia no indica que no haya posibilidades de actuarla (al menos en contra de sí mismos).

En conclusión, los trastornos psicopatológicos serios, tal como se da en los adultos, parecen implicar también un potencial autoagresivo importante en caso de ser actuado, ya que los niños con rasgos psicóticos - Grupo B - tuvieron, en promedio, más indicadores de riesgo suicida que el Grupo A y que el Grupo C, en los cuales, como en todo el resto del material, hay menores indicadores de patología.

Pareciera entonces que en estos niños habría mayor potencial suicida, en parte por las graves distorsiones de sus funciones de realidad, que les impedirían encontrar soluciones lógicas a sus problemas y, en parte por no poder descargar en el medio sus aspectos agresivos de manera adecuada, probablemente por falencias en sus interacciones. Conservarían entonces todo el poder de la conducta agresiva hacia sí mismos; el componente de culpa persecutoria (Abadi, Yampei, Rolla, A. Garma y E. Garma, 1973) que parece haber entre los suicidas, conlleva la dificultad de exteriorizar hostilidad hacia figuras de las que dependen, por lo que ésta termina revirtiendo sobre sí.

Con respecto a la edad (Passalacqua et al, 1999), la predominancia de signos alrededor de los 10 años, podría sugerir un cierto adelanto de la etapa puberal, en la que recrudece la simbiosis (García Arzeno, 1999); 
se produce una regresión a la primitiva etapa del deambulador - etapa de transición en cuanto a la separación y retorno a la madre (ansiedad de separación y satisfacción con el retorno a la figura materna) produciéndose un vacio de identidad cuando no se la encuentra disponible.

Otro observable es que el número de signos de riesgo suicida de las niñas asistidas en el Servicio de Psicología Clínica de Niños, de modo similar a los de las consultantes provenientes de otros niveles socioeconómicos, es mayor al de los varones. Esto sucede a pesar de que, comparativamente, es reducida la cantidad de derivaciones que hay entre las niñas, que merece un análisis más pormenorizado.

Esto puede deberse a que la menor expresión agresiva que, en general, ellas manifiestan, por un lado determina que pasen desapercibidas para sus maestros, poco advertidos en su mayor parte, sobre el modo de detectar psicopatología grave, particularmente cuando se trata de niños que no molestan en la clase, debido a su pasividad. Sin embargo, esto parece constituirse en un factor de riesgo mayor, en lo que se refiere al potencial suicida.

Esto se ve acentuado por la cantidad de contenidos significativos que se han encontrado en los Rorschach administrados a la población estudiada, muchos relacionados con las fantasías que son comunes en los protocolos pertenecientes a población de riesgo (Passalacqua et al, 1997), y que corresponden a las siguientes temáticas:

- Fantasías relacionadas con la muerte en general:

Néstor dijo en la L. I: "Ví una mariposa muerta, la reviví, le toqué las alas y voló. Estaba por morirse. ¡Vamos, mariposa; no te caigas, no es hora de morirse!". Adrián, en la misma lámina, dice: "Una mariposa [...] está muerta, se cayó de espaldas y se le abrió la espalda y se le ve el corazón". Axel de 6 años dice en L I: (inquieto) "Un gato" (toma la lámina y la patea); L II: "Un gato que tiene sangre, un gato muerto, sangre abajo"; L IV (se mueve, se tira al piso, toma un 
chicle del suelo y se lo mete en la boca) "Un esqueleto, es el esqueleto de un gato" (avanza sobre mí, me muerde, me arranca los anteojos y los tira al suelo).

- Fantasías relacionadas con el suicidio en especial:

Estas, tal vez por la edad de esta población, son las que menos aparecen en estos protocolos. En general se refieren a la vuelta al antro materno y al intento de huida de lo mundano, terrenal y erótico para ascender espiritualmente y encontrar paz, frialdad y expiación de culpas.

- Fantasías acerca del modo de matarse (independientemente de ser luego efectivamente el método elegido):

Adrián, varón de 8 años que puntúa 20 signos Rorschach de riesgo suicida; manifiesta explícitamente miedo a que lo abandonen y, al mismo tiempo, su intención de suicidarse. Intentó tirarse de la terraza de su casa, salvándolo su hermano, tomándolo de las piernas. Dice en la L. II: "Las alas, la sangre, se cayó y se reventó. Una paloma, viene a velocidad y se va a estrellar; son blancas las palomas; en la terraza hay rejas porque me quise tirar; me caía, me agarraba de un techo y no me pasaba nada". En la L. III: "Dos tipos que se caen del edificio, estaban pintando con rojo y se cayeron para atrás y se rompieron la cabeza". Y en L. VII: "Dos nenas que se caen, se tiraron de alguna altura".

- Fantasías relacionadas con los psicodinamismos subyacentes fundamentalmente una intensa ansiedad persecutoria (Yampei, Boronat, Graña, Rolla y Sauri, 1992; Yampei, Calesti et al., 1998): Teresa en la L VI: "Un gatito. Son así cuando se asustan o cuando quedan electrocutados. Un gatito mío que se electrocutó, quedó así”. Germán, de 10 años, que fue expulsado de la escuela por pegar a sus compañeros y a la maestra, dice en L I: "Un vampiro raro. Un monstruo, saluda para matar a alguien. La señora que va a matar le dice ¡hola! Y le tira un cuchillo”.

También se encuentran diferencias - aunque muy pequeñas - en favor de la disminución de signos de riesgo suicida en una muestra extraída 
de una población clínica de otro nivel socioeconómico (medio y medio alto), consultantes mayoritariamente por problemas de aprendizaje, con los que también se realizó la comparación $(N=23$ y Media $=12.84)$.

Las diferencias resultaron mayores al compararlos con no consultantes $(N=20$ y Media $=8.27)$, lo que señala mayor riesgo para los que se encuentran en la población de consultantes por conductas violentas pertenecientes a un medio socioeconómico medio bajo o bajo.

Esto acentúa la necesidad de continuar el arduo pero gratificante trabajo iniciado con esta población, como así también la de planificar actividades preventivas con poblaciones más amplias de similares características.

\section{Referencias}

Abadi, M., Yampei, N., Rolla, E., Garma, A. y Garma, E. (1973). La fascinación de la muerte. Buenos Aires: Paidós.

Achenbach, T. M. y Edelbrock, C. (1983). Manual for the Child Behaviour CheckList and revised Child Behaviour Profile. Burlington, V.T: University of Vermont. Department of Psychiatry.

American Psychiatric Association (1994). Manual Diagnóstico y Estadístico de los Trastornos Mentales (4 ed.). Barcelona: Masson. Bion, W. (1957). Differentiation of the Psychotic from the Non-Psychotic Personalities. En W. Bion (Ed.), Second Thoughts (pp. 43-64). Londres: Heinemann.

CEPAL/Naciones Unidas (1994). Familia y futuro. Un programa regional en América Latina y el Caribe. Santiago de Chile: autor.

Cervone, N. y Luzzi, A. M. (1996). La violencia y sus diferentes manifestaciones en los grupos terapéuticos de niños. FLAPAG (Ed.), Los vínculos en América Latina (Vol. 2) (pp. 93-100). Buenos Aires: Psinet. 
Cervone, N., Luzzi, A. M., Slapak, S. y Samaniego, C. (1999). Estudio descriptivo de una población infantil derivada para su asistencia clínica en un Centro Comunitario. Anuario 7, 203-222.

Efrom, A. M., Fainberg, E., Kleiner, Y. Sigal, A. M. y Woiskoboinik, P. (1987). La Hora de Juego Diagnóstica. En M. Siquier de Ocampo et al (Eds.), Las técnicas proyectivas y el proceso psicodiagnóstico (pp. 195-222). Buenos Aires: Nueva Visión.

García Arzeno, M. E. (1999). Entre los diez y los quince. Necesidad de aferrarse a una madre y no encontrarla. Periódico de divulgación cientifica "Uno y Otros", 26, 4-6.

Klein, M. (1952). Some theoretical conclusions regarding the emotional life of the infant. En The Melanie Klein Trust (Ed.) (1975), Melanie Klein: Envy and Gratitude and Other Works 1946-1963 (pp. 6193). Londres: The Hogart Press.

Kliksberg, B. (1997). Repensando el Estado para el desarrollo social más allá de dogmas y convencionalismos. Facultad de Ciencias Económicas, Universidad de Buenos Aires (mimeo).

Passalacqua, A., Orcoyen, D. y Herrera, M. T. (1997). Investigación sobre suicidio y Rorschach, Investigaciones en Psicología, 2 (1), 73-87. Passalacqua, A., Menestrina, N., Núñez, A. M., Simonotto, T., Borbea de Aranda, M., Pestana, L., Mussoni, A., Caro, L., Ferrari, G., Boutoure, M., Nanni, M., Piccone, A., Gaitán, L. y Pastorini, M. (1999). Observaciones sobre el Potencial Suicida en Niños. Ponencia presentada en el XVI Congreso Internacional de Rorschach y otras Técnicas Proyectivas organizado por la I.R.S, Amsterdam, Holanda.

Sautu, R., Slapak, S., Di Virgilio, M., Luzzi, A. M. y Martinez Mendoza, R. (1996). Pobreza, violencia y fracaso escolar: el papel de la familia y la escuela. V Anuario de Investigaciones. Secretaría de Investigaciones (461.480). Buenos Aires: Facultad de Psicología - UBA.

Sautu, R., Slapak, S., Di Virgilio, M., Luzzi, A. M. y; Martínez Mendoza, R. (1999, agosto). Problemas de conducta y dificultades de 
aprendizaje entre niños pobres de Buenos Aires. Revista Sociedad, 14, 79-101.

Slapak, S., Cervone, N. y Luzzi, A. M. (1998). Contención institucional en niños con conductas violentas. VI Anuario de investigaciones (pp. 440-450). Buenos Aires: Facultad de Psicología-UBA.

UNICEF (1991a). Análisis de situación. Menores en circunstancias especialmente dificiles. Uruguay. $\mathrm{N}^{\circ} 09$.

UNICEF (1991b). Análisis de situación. Menores en circunstancias especialmente dificiles. Bolivia. $\mathrm{N}^{\circ} 08$.

UNICEF (1992). Análisis de situación. Menores en circunstancias especialmente dificiles. Argentina. $\mathrm{N}^{\circ} 10$.

Winnicott, D. (1956). La tendencia antisocial. En D. Winnicott (1958) Escritos de Pediatría y Psicoanálisis 1931-1956, 413-425. Barcelona: Laia.

Yampei, N., Boronat, C., Graña, D., Rolla, D. y Sauri, J. (1992). Desesperación y suicidio. Buenos Aires: Kargieman.

Yampei, N., Calesti, T., Frigerio, R., Gravano, A., Jones, A., Sabbioni de Vega, E., Saimovici, E. y Trotta, M. (1998). Crisis y Suicidio. Grupo de Investigación sobre Crisis y Suicidio. Buenos Aires: APA. 